\title{
Controllability of switched DAEs: The single switch case
}

\author{
Markus G.-M. Ruppert and Stephan Trenn* \\ Fachbereich Mathematik, TU Kaiserslautern, 67663 Kaiserslautern
}

We study controllability of switched DAEs and formulate a definition of controllability in the behavioral sense. In order to characterize controllability for switched DAEs we first present new characterizations of controllability of non-switched DAEs based on the Wong-sequences. Afterwards a first result concerning the single-switch case is presented.

Copyright line will be provided by the publisher

\section{Controllability definition}

We study switched DAEs of the form

$$
E_{\sigma} \dot{x}=A_{\sigma} x+B_{\sigma} u
$$

where we adopt the distributional solution framework from [5]. We make the following assumptions:

1. the switching signal $\sigma: \mathbb{R} \rightarrow \mathcal{P}, \mathcal{P}$ some finite or infinite index set, is piecewise constant without accumulation of jumps;

2. each matrix pair $\left(E_{p}, A_{p}\right) \in \mathbb{R}^{n \times n} \times \mathbb{R}^{n \times n}, p \in \mathcal{P}$, is regular, i.e. $\operatorname{det}\left(s E_{p}-A_{p}\right) \not \equiv 0$.

Under these assumptions, we can define the distributional behavior

$$
\mathcal{B}_{\sigma}:=\left\{(x, u) \in \mathbb{D}_{\mathrm{pw}}^{n} \infty \times \mathbb{D}_{\mathrm{pwC}}^{m} \mid E_{\sigma} \dot{x}=A_{\sigma} x+B_{\sigma} u\right\}
$$

where $\mathbb{D}_{\text {pwC }}{ }^{\infty}$ denotes the space of piecewise-smooth distributions as defined in [5]. Due to the regularity assumption we have that $u$ is indeed an input, i.e.

$$
\forall u \in \mathbb{D}_{\mathrm{pw} \mathcal{C}^{\infty}}^{m} \exists x \in \mathbb{D}_{\mathrm{pw} \mathcal{C}^{\infty}}^{n}: \quad(x, u) \in \mathcal{B}_{\sigma}
$$

furthermore $\mathcal{B}_{\sigma}$ is a linear space, i.e. $\left(\lambda_{1} x_{1}+\lambda_{2} x_{2}, \lambda_{1} u_{1}+\lambda_{2} u_{2}\right) \in \mathcal{B}_{\sigma}$ for any $\lambda_{1}, \lambda_{2} \in \mathbb{R}$ and $\left(x_{1}, u_{1}\right),\left(x_{2}, u_{2}\right) \in \mathcal{B}_{\sigma}$.

Definition 1.1 (Controllability) We call (1) controllable (from $t=0$ onwards) if, and only if, $\mathcal{B}_{\sigma}$ is controllable in the behavioral sense, i.e.

$$
\begin{aligned}
& \forall\left(x_{1}, u_{1}\right),\left(x_{2}, u_{2}\right) \in \mathcal{B}_{\sigma} \exists T \geq 0 \exists\left(x_{12}, u_{12}\right) \in \mathcal{B}_{\sigma}: \\
&\left(x_{12}, u_{12}\right)_{(-\infty, 0)}=\left(x_{1}, u_{1}\right)_{(-\infty, 0)} \wedge\left(x_{12}, u_{12}\right)_{(T, \infty)}=\left(x_{2}, u_{2}\right)_{(T, \infty)}
\end{aligned}
$$

Lemma 1.2 (Controllability to zero) The switched DAE (1) is controllable if, and only if, any feasible trajectory can be controlled to zero, i.e.

$$
\forall(x, u) \in \mathcal{B}_{\sigma} \exists T \geq 0 \exists\left(x_{0}, u_{0}\right) \in \mathcal{B}_{\sigma}: \quad\left(x_{0}, u_{0}\right)_{(-\infty, 0)}=(x, u)_{(-\infty, 0)} \wedge\left(x_{0}, u_{0}\right)_{(T, \infty)}=(0,0)
$$

Proof. Since $\left(x_{2}, u_{2}\right)=(0,0) \in \mathcal{B}_{\sigma}$ necessity is clear. For the converse let $\left(x_{1}, u_{1}\right),\left(x_{2}, u_{2}\right) \in \mathcal{B}_{\sigma}$ and choose $\left(x_{0}, u_{0}\right) \in$ $\mathcal{B}_{\sigma}$ such that $\left(x_{0}, u_{0}\right)_{(-\infty, 0)}=\left(x_{1}-x_{2}, u_{1}-u_{2}\right)_{(-\infty, 0)}$ and $\left(x_{0}, u_{0}\right)_{(T, \infty)}=0$ for some $T \geq 0$. Now $\left(x_{12}, u_{12}\right):=$ $\left(x_{0}, u_{0}\right)+\left(x_{2}, u_{2}\right)$ satisfies $\left(x_{12}, u_{12}\right)_{(-\infty, 0)}=\left(x_{1}, u_{1}\right)_{(-\infty, 0)}$ and $\left(x_{12}, u_{12}\right)_{(T, \infty)}=\left(x_{2}, u_{2}\right)_{(T, \infty)}$.

Hence in the following we can restrict our attention to the question whether it is possible to steer any past trajectory of (1) to zero.

* Corresponding author: e-mail trenn@mathematik.uni-kl.de, phone +49 6312054134 


\section{Preliminary results for nonswitched DAEs}

Consider the DAE

$$
E \dot{x}=A x+B u
$$

with regular matrix pair $(E, A)$. For the analysis of (2) the Wong-sequences [7] proved themselves extremely helpful:

$$
\mathcal{V}_{(E, A)}^{0}:=\mathbb{R}^{n}, \quad \mathcal{V}_{(E, A)}^{i+1}:=A^{-1}\left(E \mathcal{V}_{(E, A)}^{i}\right), \quad \mathcal{W}_{(E, A)}^{0}:=\{0\}, \quad \mathcal{W}^{i+1}:=E^{-1}\left(A \mathcal{W}_{(E, A)}^{i}\right), \quad i=0,1,2, \ldots
$$

Both Wong sequences are nested and consequently get stationary after finitely many steps, let

$$
\mathcal{V}_{(E, A)}^{*}:=\bigcap_{i \in \mathbb{N}} \mathcal{V}_{(E, A)}^{i}=\mathcal{V}_{(E, A)}^{i^{*}}, \quad \mathcal{W}_{(E, A)}^{*}=\bigcup_{i \in \mathbb{N}} \mathcal{W}_{(E, A)}^{i}=\mathcal{W}_{(E, A)}^{i^{*}}
$$

for some suitable $i^{*} \in \mathbb{N}$. We have the following important result

Theorem 2.1 ( [1]) Let $\mathcal{V}_{(E, A)}^{*}$ and $\mathcal{W}_{(E, A)}^{*}$ be the limits of the Wong sequences (3) for a regular matrix pair (E,A) and let $V$ and $W$ be full column rank matrices such that $\operatorname{im} V=\mathcal{V}_{(E, A)}^{*}$ and $\operatorname{im} W=\mathcal{W}_{(E, A)}^{*}$. Then $T:=[V, W]$ and $S:=[E V, A W]^{-1}$ are invertible matrices and transform $(E, A)$ into the quasi-Weierstrass form $(Q W F)$

$$
(S E T, S A T, S B)=\left(\left[\begin{array}{cc}
I & 0 \\
0 & N
\end{array}\right],\left[\begin{array}{ll}
J & 0 \\
0 & I
\end{array}\right],\left[\begin{array}{l}
B_{1} \\
B_{2}
\end{array}\right]\right)
$$

where $J \in \mathbb{R}^{n_{1} \times n_{1}}, 0 \leq n_{1} \leq n$, is some matrix and $N \in \mathbb{R}^{n_{2} \times n_{2}}, n_{2}:=n-n_{1}$, is nilpotent.

Definition 2.2 (Consistency, differential and impulse projector) With the notation of Theorem 2.1 define the consistency, the differential and the impulse projector resp.

$$
\Pi_{(E, A)}:=T\left[\begin{array}{ll}
I & 0 \\
0 & 0
\end{array}\right] T^{-1}, \quad \Pi_{(E, A)}^{\text {diff }}:=T\left[\begin{array}{ll}
I & 0 \\
0 & 0
\end{array}\right] S, \quad \Pi_{(E, A)}^{\mathrm{imp}}:=T\left[\begin{array}{ll}
0 & 0 \\
0 & I
\end{array}\right] S,
$$

where the block matrix sizes correspond to the block sizes in the QWF. Furthermore, let

$$
A^{\text {diff }}:=\Pi^{\text {diff }} A, \quad B^{\text {diff }}:=\Pi^{\text {diff }} B, \quad E^{\text {imp }}:=\Pi^{\text {imp }} E, \quad B^{\text {imp }}:=\Pi^{\text {imp }} B .
$$

Note that the differential and impulse projectors are not idempotent and hence are not projectors in the usual sense.

The set of consistent initial values for (2)

$$
\mathcal{V}_{(E, A, B)}^{*}:=\left\{x_{0} \in \mathbb{R}^{n} \mid \exists \text { smooth solution }(x, u) \text { of }(2) \text { with } x(0)=x_{0}\right\}
$$

can now be given as follows:

Lemma 2.3

$$
\mathcal{V}_{(E, A, B)}^{*}=\mathcal{V}_{(E, A)}^{*} \oplus \operatorname{im}\left\langle E^{\mathrm{imp}}, B^{\mathrm{imp}}\right\rangle
$$

where $\langle M, P\rangle:=\left[P, M P, M^{2} P, \ldots M^{n-1} P\right]$ for matrices $M \in \mathbb{R}^{n \times n}, P \in \mathbb{R}^{n \times m}$.

The proof is omitted due to space limitations.

Remark 2.4 The space $\mathcal{V}_{(E, A, B)}^{*}$ can itself be expressed as the limit of the first augmented Wong sequence [2]

$$
\mathcal{V}_{(E, A, B)}^{0}=\mathbb{R}^{n}, \quad \mathcal{V}_{(E, A, B)}^{i+1}=A^{-1}\left(E \mathcal{V}_{(E, A, B)}^{i}+\operatorname{im} B\right), \quad i=0,1,2, \ldots,
$$

which establishes a nice connection between the first Wong sequence and the augmented first Wong sequence. It can also be shown [3] that the following connection holds:

$$
\mathcal{W}_{(E, A, B)}^{*}=\mathcal{W}_{(E, A)}^{*} \oplus \operatorname{im}\left\langle A^{\text {diff }}, B^{\text {diff }}\right\rangle,
$$

where $\mathcal{W}_{(E, A, B)}^{*}$ is the limit of the second augmented Wong sequence

$$
\mathcal{W}_{(E, A, B)}^{0}=\{0\}, \quad \mathcal{W}_{(E, A, B)}^{i+1}=E^{-1}\left(A \mathcal{W}_{(E, A, B)}^{i}+\operatorname{im} B\right), \quad i=0,1,2, \ldots
$$

It is a well known fact (see e.g. [2]) that controllability of (2) in the behavioral sense is equivalent to R-controllability in the sense of [4] which in turn is equivalent to the controllability of the ODE $\dot{v}=J v+B_{1} u$. In fact, it is possible to characterize controllability in terms of the original system matrices and the above projectors: 


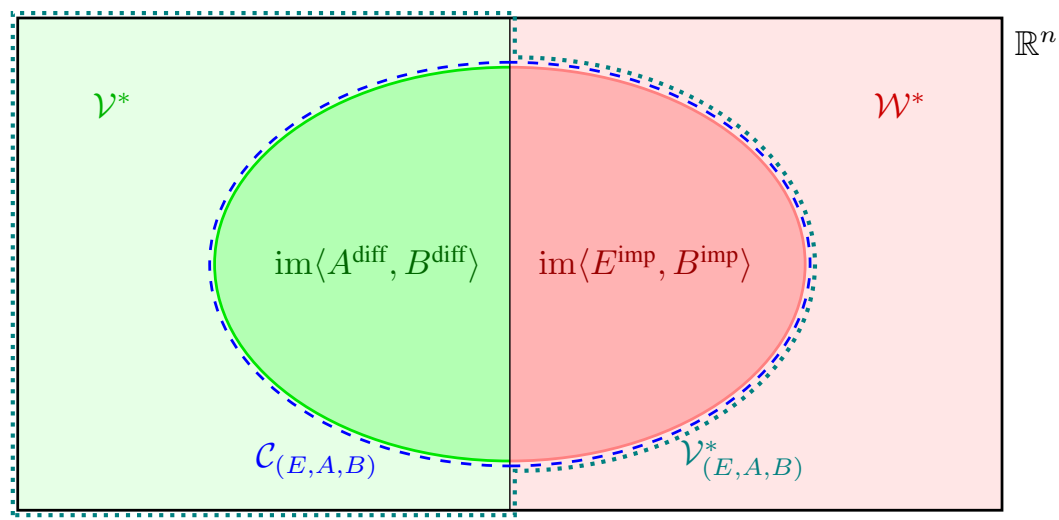

Fig. 1: The relationship between the spaces $\mathcal{V}^{*}, \mathcal{W}^{*}, \operatorname{im}\left\langle A^{\text {diff }}, B^{\text {diff }}\right\rangle, \operatorname{im}\left\langle E^{\text {imp }}, B^{\text {imp }}\right\rangle$.

Lemma 2.5 Consider the DAE (2) with regular matrix pair $(E, A)$ and the notation from Definition 2.2. The controllability space of (2) given by $\mathcal{C}_{(E, A, B)}:=\left\{x_{0} \in \mathbb{R}^{n} \mid \exists\right.$ solution $\left.(x, u) \exists T \geq 0: \quad x(0)=x_{0} \wedge x(T)=0\right\}$ satisfies

$$
\mathcal{C}_{(E, A, B)}=\mathcal{V}_{(E, A, B)}^{*} \cap \mathcal{W}_{(E, A, B)}^{*}=\operatorname{im}\left\langle A^{\text {diff }}, B^{\text {diff }}\right\rangle \oplus \operatorname{im}\left\langle E^{\text {imp }}, B^{\text {imp }}\right\rangle .
$$

Pro of. Assume first the DAE (2) is in QWF, then we have that the controllability space is given by $\operatorname{im}\left\langle J, B_{1}\right\rangle \times \operatorname{im}\left\langle N, B_{2}\right\rangle$. With a simple rewriting we obtain the claim.

The different subspace relationships are illustrated in Figure 1.

We conclude the section with a generalized Kalman controllability decomposition:

Theorem 2.6 (Quasi-Weierstrass Kalman Controllability Decomposition) Consider a regular DAE (2) with corresponding projectors as in Definition 2.2. Choose full column rank matrices $T_{1}, T_{2}, T_{3}, T_{4}$ as follows:

$$
\operatorname{im} T_{1}=\operatorname{im}\left\langle A^{\text {diff }}, B^{\text {diff }}\right\rangle, \quad \operatorname{im} T_{1} \oplus \operatorname{im} T_{2}=\mathcal{V}^{*}, \quad \operatorname{im} T_{3}=\operatorname{im}\left\langle E^{\text {imp }}, B^{\text {imp }}\right\rangle, \quad \operatorname{im} T_{3} \oplus \operatorname{im} T_{4}=\mathcal{W}^{*}
$$

Then $T:=\left[T_{1}, T_{2}, T_{3}, T_{4}\right]$ and $S:=\left[E T_{1}, E T_{2}, A T_{3}, A T_{4}\right]$ transform $(E, A, B)$ into the quasi-Weierstrass Kalman controllability decomposition $(Q W K C D)$.

$$
(S E T, S A T, S B)=\left(\left[\begin{array}{cccc}
I & 0 & 0 & 0 \\
0 & I & 0 & 0 \\
0 & 0 & N_{1} & N_{2} \\
0 & 0 & 0 & N_{3}
\end{array}\right],\left[\begin{array}{cccc}
J_{1} & J_{2} & 0 & 0 \\
0 & J_{3} & 0 & 0 \\
0 & 0 & I & 0 \\
0 & 0 & 0 & I
\end{array}\right],\left[\begin{array}{c}
B_{J} \\
0 \\
B_{N} \\
0
\end{array}\right]\right),
$$

where the DAE corresponding to

$$
\left(E_{C}, A_{C}, B_{C}\right):=\left(\left[\begin{array}{cc}
I & 0 \\
0 & N_{1}
\end{array}\right],\left[\begin{array}{cc}
J_{1} & 0 \\
0 & I
\end{array}\right],\left[\begin{array}{c}
B_{J} \\
B_{N}
\end{array}\right]\right)
$$

is completely controllable, i.e. $\mathcal{C}_{\left(E_{C}, A_{C}, B_{C}\right)}=\mathbb{R}^{n_{1}+n_{3}}$ where $n_{i} \in \mathbb{N}$ denotes the number of columns in $T_{i}$.

The proof is omitted due to space limitations.

\section{The single switch case}

Consider the switched DAE (1) with the switching signal

$$
\sigma_{1}(t):= \begin{cases}1, & t<\varepsilon, \\ 2, & t \geq \varepsilon .\end{cases}
$$

Proposition 3.1 (Sufficient condition for controllability) The switched DAE (1) with switching signal $\sigma_{1}$ is controllable if

$$
\operatorname{im}\left\langle A_{1}^{\text {diff }}, B_{1}^{\text {diff }}\right\rangle+\Pi_{\left(E_{2}, A_{2}\right)}^{-1} \operatorname{im}\left\langle A_{2}^{\text {diff }}, B_{2}^{\text {diff }}\right\rangle \supseteq \mathcal{V}_{\left(E_{1}, A_{1}\right)}^{*} .
$$

Proof. We have to show that for any consistent initial value $x_{0} \in \mathcal{V}_{\left(E_{1}, A_{1}, B_{1}\right)}^{*}$ there exists an input $u$ and a time $t_{1}>\varepsilon$ such that the corresponding solution $x$ satisfies $x\left(t_{1}\right)=0$. Note that adding $\operatorname{im}\left\langle E_{1}^{\mathrm{imp}}, B_{1}^{\mathrm{imp}}\right\rangle$ on both sides of (6) implies (invoking Lemmas 2.3 and 2.5)

$$
\mathcal{V}_{\left(E_{1}, A_{1}, B_{1}\right)}^{*} \subseteq \mathcal{C}_{\left(E_{1}, A_{1}, B_{1}\right)}+\Pi_{\left(E_{2}, A_{2}\right)}^{-1} \operatorname{im}\left\langle A_{2}^{\text {diff }}, B_{2}^{\text {diff }}\right\rangle .
$$


Denote with $\varphi\left(\cdot, x_{0}, u\right)$ the (unique) distributional solution of (1) with switching signal $\sigma_{1}$ with initial condition $x(0-)=x_{0} \in$ $\mathcal{V}_{\left(E_{1}, A_{1}, B_{1}\right)}^{*}$ and input $u$. Then $\varphi\left(t-, x_{0}, 0\right) \in \mathcal{V}_{\left(E_{1}, A_{1}, B_{1}\right)}^{*}$ for all $t \in(0, \varepsilon)$. Invoking (7) we may choose $x_{1} \in \mathcal{C}_{\left(E_{1}, A_{1}, B_{1}\right)}$ such that $\varphi\left(\varepsilon-, x_{0}, 0\right)-x_{1} \in \Pi_{\left(E_{2}, A_{2}\right)}^{-1} \operatorname{im}\left\langle A_{2}^{\text {diff }}, B_{2}^{\text {diff }}\right\rangle$. Since $x_{1} \in \mathcal{C}_{\left(E_{1}, A_{1}, B_{1}\right)}$ we can choose $u$ (specified on $\left.[0, \varepsilon)\right)$ such that $\varphi(\varepsilon-, 0, u)=-x_{1}$. Due to linearity, the solution $x$ starting at $x_{0}$ and with input $u$ then satisfies:

$$
x(\varepsilon-)=\varphi\left(\varepsilon-, x_{0}, 0\right)+\varphi(\varepsilon-, 0, u)=\varphi\left(\varepsilon-, x_{0}, 0\right)-x_{1} \in \Pi_{\left(E_{2}, A_{2}\right)}^{-1} \operatorname{im}\left\langle A_{2}^{\text {diff }}, B_{2}^{\text {diff }}\right\rangle .
$$

Letting $u^{(i)}(\varepsilon+)=0$ for all $i \in \mathbb{N}$ we know

$$
x(\varepsilon+)=\Pi_{\left(E_{2}, A_{2}\right)} x(\varepsilon-) \in \operatorname{im}\left\langle A_{2}^{\text {diff }}, B_{2}^{\text {diff }}\right\rangle \subseteq \mathcal{C}_{\left(E_{2}, A_{2}, B_{2}\right)} .
$$

Hence we can find $t_{1}>\varepsilon$ and $u$ further specified on $\left(\varepsilon, t_{1}\right)$ such that the solution satisfies $x\left(t_{1}-\right)=0$.

Remark 3.2 Note that $\operatorname{im}\left\langle E_{2}^{\mathrm{imp}}, B_{2}^{\mathrm{imp}}\right\rangle \subseteq \mathcal{W}_{\left(E_{2}, A_{2}\right)}^{*}$ and $\Pi_{\left(E_{2}, A_{2}\right)}^{-1} \mathcal{W}_{\left(E_{2}, A_{2}\right)}^{*}=\{0\}$. Hence

$$
\Pi_{\left(E_{2}, A_{2}\right)}^{-1} \operatorname{im}\left\langle A_{2}^{\text {diff }}, B_{2}^{\text {diff }}\right\rangle=\Pi_{\left(E_{2}, A_{2}\right)}^{-1} \mathcal{C}_{\left(E_{2}, A_{2}, B_{2}\right)} .
$$

Hence (6) implies

$$
\mathcal{C}_{\left(E_{1}, A_{1}, B_{1}\right)}+\Pi_{\left(E_{2}, A_{2}\right)}^{-1} \mathcal{C}_{\left(E_{2}, A_{2}, B_{2}\right)} \supseteq \mathcal{V}_{\left(E_{1}, A_{1}, B_{1}\right)}^{*},
$$

i.e. any consistent initial values can be written as the sum of two parts, the first part corresponds to an initial value which can be controlled to zero before the switch and the second part corresponds to a value can be controlled to zero after the switch. However, condition (8) is not equivalent to (6) as the following example ${ }^{1}$ shows:

$$
E_{1}=\left[\begin{array}{ll}
1 & 0 \\
0 & 0
\end{array}\right], A_{1}=\left[\begin{array}{ll}
0 & 0 \\
0 & 1
\end{array}\right], B_{1}=\left[\begin{array}{l}
0 \\
1
\end{array}\right], \quad E_{2}=\left[\begin{array}{ll}
1 & 0 \\
0 & 1
\end{array}\right], A_{2}=\left[\begin{array}{ll}
0 & 0 \\
0 & 0
\end{array}\right], B_{2}=\left[\begin{array}{l}
1 \\
1
\end{array}\right] .
$$

It is easily seen that

$$
\mathcal{V}_{\left(E_{1}, A_{1}\right)}^{*}=\mathbb{R} \times\{0\}, \operatorname{im}\left\langle A_{1}^{\text {diff }}, B_{1}^{\text {diff }}\right\rangle=\{0\}^{2}, \operatorname{im}\left\langle A_{2}^{\text {diff }}, B_{2}^{\text {diff }}\right\rangle=\operatorname{im}\left[\begin{array}{l}
1 \\
1
\end{array}\right], \Pi_{\left(E_{2}, A_{2}\right)}=I .
$$

Hence condition (6) is not fulfilled as $\operatorname{im}\left[\begin{array}{l}1 \\ 1\end{array}\right] \nsubseteq \mathbb{R} \times\{0\}$. However,

$$
\mathcal{C}_{\left(E_{1}, A_{1}, B_{1}\right)}=\{0\} \times \mathbb{R} \quad \text { and hence } \quad \mathcal{C}_{\left(E_{1}, A_{1}, B_{1}\right)}+\operatorname{im}\left[\begin{array}{l}
1 \\
1
\end{array}\right]=\mathbb{R}^{2}
$$

and hence condition (8) is satisfied. In particular, condition (6) is not a necessary condition for controllability of the switched system and it remains a topic of future research to investigate whether condition (8) is in fact a characterization of controllability.

\section{References}

[1] Thomas Berger, Achim Ilchmann, and Stephan Trenn. The quasi-Weierstraß form for regular matrix pencils. Lin. Alg. Appl., 436(10):4052-4069, 2012.

[2] Thomas Berger and Timo Reis. Controllability of linear differential-algebraic systems - a survey. In Achim Ilchmann and Timo Reis, editors, Surveys in Differential-Algebraic Equations I, Differential-Algebraic Equations Forum, pages 1-61. Springer-Verlag, BerlinHeidelberg, 2013.

[3] Thomas Berger and Stephan Trenn. Kalman controllability decompositions for differential-algebraic systems. Syst. Control Lett., 2014. conditionally accepted.

[4] Liyi Dai. Singular Control Systems. Number 118 in Lecture Notes in Control and Information Sciences. Springer-Verlag, Berlin, 1989.

[5] Stephan Trenn. Distributional differential algebraic equations. PhD thesis, Institut für Mathematik, Technische Universität Ilmenau, Universitätsverlag Ilmenau, Ilmenau, Germany, 2009.

[6] Stephan Trenn. Switched differential algebraic equations. In Francesco Vasca and Luigi Iannelli, editors, Dynamics and Control of Switched Electronic Systems - Advanced Perspectives for Modeling, Simulation and Control of Power Converters, chapter 6, pages 189-216. Springer-Verlag, London, 2012.

[7] Kai-Tak Wong. The eigenvalue problem $\lambda T x+$ Sx. J. Diff. Eqns., 16:270-280, 1974.

\footnotetext{
${ }^{1}$ We thank Ferdinand Küsters for making us aware of this example
} 